Vietnam Journal of Mechanics, VAST, Vol.39, No. 4 (2017), pp. $339-349$

DOI:10.15625/0866-7136/9714

\title{
AN IMPROVED NUMERICAL METHOD FOR A 2D POLLUTION WATER MODEL: DIRECT MODEL
}

\author{
Nguyen Hong Phong ${ }^{1,2}$, Tran Thu Ha ${ }^{1, *}$, F. X. Le Dimet ${ }^{3}$, Duong Ngoc Hai ${ }^{1}$ \\ ${ }^{1}$ Institute of Mechanics, VAST, 18 Hoang Quoc Viet, Hanoi, Vietnam \\ ${ }^{2}$ VNU University of Engineering and Technology, Hanoi, Vietnam \\ ${ }^{3}$ Laboratoire Jean-Kunzmann, Saint Martin d'Hères, France \\ *E-mail: ttha@imech.vast.vn \\ Received April 25, 2017
}

\begin{abstract}
In this paper a 2D pollution water model with an improved numerical method is considered. In order to reduce the approximation errors of the numerical scheme, a new approximation method is introduced to calculate the concentration flux between two cells ( $j$-cell and $l$-cell) in the direction of the normal vector $\vec{n}$ orthogonal to their common side. The advantage of this approximation is that the concentration flux $\partial C / \partial \vec{n}$ from $j$-cell to $l$-cell and the other one from $l$-cell to $j$-cell are only different by their signs but not by their absolute values. Therefore, the errors of concentration simulated by this scheme are reduced and less than those obtained by a normal differential implicit discretization. This improvement of the scheme will be illustrated by two test cases. In the numerical tests we will display the difference between the exact solution, the classical scheme and the proposed scheme. Numerical results demonstrate the improvement of this approach.
\end{abstract}

Keywords: Water pollution, finite volume method.

\section{INTRODUCTION}

Water pollution is one of the most serious problems worldwide. A major scientific challenge is the ability to predict the evolution of an episode of pollution. To achieve this goal we need to mix several sources of information:

- Models based on equation of conservation;

- Mathematical method to solve the above models;

- Observations in situ or remote measurements;

- Statistics on the fields;

- Images.

Using Variational Data Assimilation will permit to retrieve the state of the flow. It is clear that the quality of the prediction takes into account the quality of the items listed above. The purpose of this paper is to present a global example in the case of water pollution. We will consider an improved model for water pollution which is used to

(c) 2017 Vietnam Academy of Science and Technology 
simulate the flow of water and the evolution of a passive contaminant. For this problem, many numerical methods and models that have been studied and developed such as WASP, QUAL2K [1] ... Based on the hydraulic model IMECH_2DUSZ [2,3] a 2D water pollution model is developed then numerically solved due to a finite volume the directions of the normal vector common to two elements are different. It leads to a numerical solution closer to the exact solution than the one obtained by using the former methods. The improvement of the model is demonstrated on two test cases for which the analytical solutions are known.

\section{DERIVING A 2D WATER POLLUTION MODEL}

The used model is a system coupling the hydraulic component and equation of concentration for the pollutant. The hydraulic model is based on the Saint-Venant equations in a domain $\Omega$ with boundary $\Gamma$ (see $[1,4])$

$$
\begin{aligned}
& \frac{\partial h}{\partial t}+\frac{\partial u h}{\partial x}+\frac{\partial u h}{\partial y}=0, \text { in } \Omega, \\
& \frac{\partial u}{\partial t}+u \frac{\partial u}{\partial x}+v \frac{\partial u}{\partial y}+g \frac{\partial h}{\partial x}=-g \frac{u\left(u^{2}+v^{2}\right)^{1 / 2}}{K_{x}^{2} h^{4 / 3}}-g \frac{\partial z_{b}}{\partial x}, \text { in } \Omega, \\
& \frac{\partial v}{\partial t}+u \frac{\partial v}{\partial x}+v \frac{\partial v}{\partial y}+g \frac{\partial h}{\partial y}=-g \frac{v\left(u^{2}+v^{2}\right)^{1 / 2}}{K_{x}^{2} h^{4 / 3}}-g \frac{\partial z_{b}}{\partial y}, \text { in } \Omega,
\end{aligned}
$$

where $\Omega$ is a bounded domain of $\mathbb{R}^{2}$ with boundary $\Gamma ; h=z-z_{b}$ is the water depth; $z$ is the free surface elevation; $z_{b}$ is the bottom elevation; $u$ is the velocity in the $x$-direction; $v$ is the velocity in the $y$-direction; $g$ is the gravity acceleration; $K_{x}$ is the Strickler coefficient in the $x$-direction; $K_{y}$ is the Strickler coefficient in the $y$-direction. We suppose that there are some passive substances dissolved in the flow. Then the transport and diffusion processes of the pollutant are governed by the following equation (see [1-6])

$$
\frac{\partial C}{\partial t}+U \vec{\nabla} C-\eta \Delta C=K C+\Phi, \text { in } \Omega .
$$

Here $\Delta=\left(\frac{\partial^{2}}{\partial x^{2}}+\frac{\partial^{2}}{\partial y^{2}}\right) ; \vec{\nabla}=\left(\frac{\partial}{\partial x}, \frac{\partial}{\partial y}\right) ; C$ is the concentration of the substance; $F$ is the source term of the pollutant; $h$ is the diffusion coefficient of the pollutant; $U=(u ; v)$ is the water velocity vector; $K$ is the conversion coefficient. The boundary $\Gamma$ is defined by $\Gamma=\Gamma_{1} \cup \Gamma_{2} \cup S_{w} . \Gamma_{1}, \Gamma_{2}, S_{w}$ are, respectively, the outflow, inflow boundaries and solid wall of the $\Omega$ domain. $\vec{n}=\left(n_{x}, n_{y}\right)$ is the unit normal vector to the inflow $\Gamma_{1}$ and outflow $\Gamma_{2}$. For a water pollution problem with a slow evolution the boundary conditions are: $U(x, y, t) \vec{n}=\bar{U}_{i n}(t)$ and $C(x, y, t)=C_{i n}(t)$ on $\Gamma_{1} ; h(x, y, t)=\bar{h}_{\text {in }}(t)$ on $\Gamma_{2} ; U(x, y, t) \vec{n}=0, \frac{\partial C(x, y, t)}{\partial \vec{n}}=0$ on the solid wall $S_{w}$. On $\Gamma_{2}$ one of the following two boundary conditions is chosen: either $\partial C / \partial \vec{n}=0$ or $C=C_{\text {out }}(t)$. Let $X$ be $(h ; u ; v)^{T}$, with a given initial conditions: $X_{0}=(h(x, y, 0), u(x, y, 0), v(x, y, 0))^{T}, U_{0}=$ $(u(x, y, 0), v(x, y, 0))^{T}$ and $C(x, y, t)$ with $C(x, y, 0)=C_{0}(x, y)$ a solution of the system is uniquely determined on $\Omega$ (see [7]). 


\section{NUMERICAL METHOD}

To compute a numerical solution of the above equations, a cell centered finite volume method is carried out (see [8]). The mesh defined on $\Omega$ is an unstructured triangulation. We divide $\Omega$, with $N_{p}$ mesh points, into $N$ cells $\Omega_{j}$ with respective boundary $\gamma_{j}$, $(j=1, \ldots, N)$. A basic cell $\Omega_{j}$ is described by left side of Fig. 1 with the index points $\left(x_{j k}, y_{j k}\right)$, center point $\left(x_{j c} y_{j c}\right)$ and the unit normal vectors $\vec{n}_{j, i k}=\left(n_{j, i k}^{x}, n_{j, i k}^{y}\right)$ to the sides $l_{j, i k}$ connecting 2 points $\left(x_{j i}, y_{j i}\right)$ and $\left(x_{j k} y_{j k}\right),(i, k=1, \ldots, 3$ with $i \neq k)$. The length of a side of a triangle is denoted by $\vec{l}_{j, i k}$.
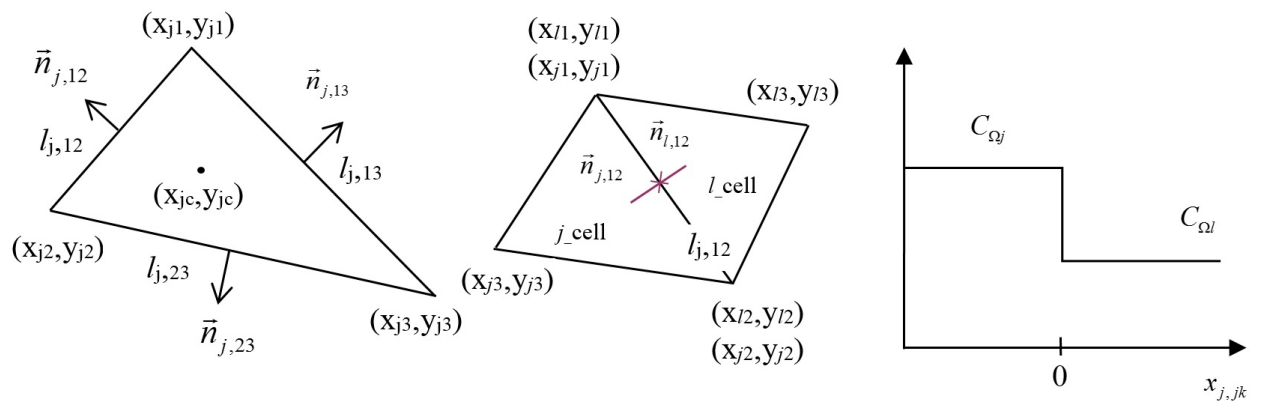

Fig. 1. A cell (left); Normal vectors to a common side between two cells (middle); Exchanging concentration between 2 cells $\Omega_{j}$ and $\Omega_{l}$ (right)

The value transfers of some functions $h, u, v, C$ from points to elements are carried out according to the following scheme for some function $w$ :

- The value of a function $w$ at the center of the cell $j$ and at a point $m\left(m=1, \ldots, N_{p}\right)$ are denoted by $w^{(j, c)}$ and $w_{m}$. The value of function $w$ at a point $m$ is presented in [3].

$$
w_{m}=\frac{1}{M} \sum_{q=1}^{M} w^{\left(j_{q}, c\right)},
$$

where $w^{\left(j_{q}, c\right)}(q=1, \ldots, M)$ are the values of function $w$ at $M$ cells that is connected to the point $m$.

- In $j$-cell we denote $w_{m_{k}}$ the value of function $w$ at the point number $m_{k}$ of coordinates $\left(x_{j k}, y_{j k}\right)\left(m_{k}=1, \ldots, N_{p}\right.$ and $\left.k=1,2,3\right)$. On this cell the approximations of partial derivatives $\partial w / \partial x$ and $\partial w / \partial y$ are introduced in [3].

$$
\begin{aligned}
& \frac{\partial w}{\partial x}=\frac{\partial w^{(j, c)}}{\partial x}=\frac{\left(w_{m 2}-w_{m 1}\right)\left(y_{j 3}-y_{j 1}\right)-\left(w_{m 3}-w_{m 1}\right)\left(y_{j 2}-y_{j 1}\right)}{\left(x_{j 2}-x_{j 1}\right)\left(y_{j 3}-y_{j 1}\right)-\left(x_{j 3}-x_{j 1}\right)\left(y_{j 2}-y_{j 1}\right)}, \\
& \frac{\partial w}{\partial y}=\frac{\partial w^{(j, c)}}{\partial y}=\frac{\left(w_{m 3}-w_{m 1}\right)\left(x_{j 2}-x_{j 1}\right)-\left(w_{m 2}-w_{m 1}\right)\left(x_{j 3}-x_{j 1}\right)}{\left(x_{j 2}-x_{j 1}\right)\left(y_{j 3}-y_{j 1}\right)-\left(x_{j 3}-x_{j 1}\right)\left(y_{j 2}-y_{j 1}\right)} .
\end{aligned}
$$

The new approximations of the functions $C$ and $\frac{\partial C}{\partial \vec{n}}$ on a common side between 2 elements (see Section 3.1) are introduced for the following reason: 
Denote $l_{j, 12}$ the common side between $j$-cell and $l$-cell (see the middle of Fig. 1 ) and $\vec{n}_{j, 12}, \vec{n}_{l, 12}$ the normal vectors to $l_{j, 12}$ directed from $j$-cell to $l$-cell and from $l$-cell to $j$-cell, respectively would be

$$
\frac{\partial C_{\Omega j}}{\partial \vec{n}_{j, 12}}=\frac{\partial C_{\Omega j}}{\partial x} n_{j, 12}^{x}+\frac{\partial C_{\Omega j}}{\partial y} n_{j, 12}^{y} \text { and } \frac{\partial C_{\Omega_{l}}}{\partial \vec{n}_{l, 12}}=\frac{\partial C_{\Omega_{l}}}{\partial x} n_{l, 12}^{x}+\frac{\partial C_{\Omega_{l}}}{\partial y} n_{l, 12}^{y} .
$$

Using the discrete formulas (4) for $j$-cell and $l$-cell (see the middle of Fig. 1) we can see that, on the common side $l_{j, 12}$, the values $\left|\frac{\partial C_{\Omega j}}{\partial \vec{n}_{j, 12}}\right|$ and $\left|\frac{\partial C_{\Omega l}}{\partial \vec{n}_{l, 12}}\right|$ may not be equal. For this reason, in order to reduce approximation errors of simulation process we will calculate the concentration function $C$ and its flux function $\frac{\partial C}{\partial \vec{n}_{j, i k}}$ on the common side $l_{j, i k}$ with $i, k=1, \ldots, 3$ and $i \neq k$ by another approximation method that will be described in Section 3.1. The 2D shallow water equations is solved by using the HLL Riemann Solver scheme (the approximate Riemann solver proposed by Harten Lax and van Leer in 1983) (see [9-11]). In this paper we present the improved method solving the concentration equation.

\subsection{Numerical method for solving the concentration equation}

To solve the concentration equation (2) we will improve the approximation scheme in such a way so that the simulation is stable and closer to the exact solutions.

Using a finite volume discretization $\Omega_{j}$ (see [3]), integrating (2) by $\Omega_{j}$ we obtain the following approximation

$$
C_{\Omega_{j, t+\Delta t}}=\frac{1}{1-0.5 K \Delta t}\left[\left(1+\frac{K \Delta t}{2}+\vec{\nabla} U_{\Omega j} \Delta t\right) C_{\Omega_{j, t}}+\Delta t \Phi+\frac{\Delta t}{\Delta S} \sum_{i, k=1, i \neq k}^{3}\left[G\left(C_{t}\right)_{l_{j, i k}} \vec{n}_{j, i k} \bar{l}_{j, i k}\right]\right],
$$

where

$$
G\left(C_{t}\right)_{l_{j, i k}} \vec{n}_{j, i k}=\left.\widehat{U}_{l_{j, i k}} C_{t}\right|_{l_{j, i k}}-\left.\eta \frac{\partial C_{t}}{\partial \vec{n}_{j, i k}}\right|_{l_{j, i k}} .
$$

On the common side $l_{j, i k}$ the function $\widehat{U}_{j, i k}=\left.U \vec{n}_{j, i k}\right|_{j, i k}$ is approximated by (see [9-11])

$$
\widehat{U}_{l_{j, i k}}=\frac{1}{2}\left(U_{\Omega_{j}}+U_{\Omega_{l}}\right) \vec{n}_{j, i k}+\sqrt{g h_{\Omega_{j}}}-\sqrt{g h_{\Omega_{l}}}
$$

Applying the ideas of Godunov and Roes Riemann Solver methods (see $[5,12,13]$ ) we estimate the value of $G\left(C_{t}\right)_{l_{j, i k}} \vec{n}_{j, i k}$ on $l_{j, i k}$. We will simulate the values of $C$ and $\frac{\partial C}{\partial \vec{n}_{j, i k}}$ by the direction of

$$
x_{j, i k} \equiv \vec{n}_{j, i k}
$$

Local coordinates $x_{j, i k}$ are set to position 0 as the contact boundary between $j$-cell and $l$-cell. The exchanged concentration $C$ and $\frac{\partial C}{\partial x_{j, i k}}$ from $\Omega_{j}$ to $\Omega_{l}$ are described in the 
right side of Fig. 1. To evaluate $C$ and $\frac{\partial C}{\partial x_{j, i k}} l_{j, i k}$ we consider the numerical solution of a 1D Riemann problem for the flux of concentration in the direction of $x_{j, i k}$ (see $\left.[5,12,13]\right)$

$$
\left\{\begin{array}{c}
\frac{\partial C}{\partial t}+\widehat{U}_{l_{j, i k}} \frac{\partial C}{\partial x_{j, i k}}-\eta \frac{\partial^{2} C}{\partial x_{j, i k}^{2}}-K C=\Phi \\
\lim _{x_{j, i k} \rightarrow \pm 0} C=\left\{\begin{array}{l}
C_{\Omega j}, x_{j, i k}<0 \\
C_{\Omega_{l}}, x_{j, i k}>0
\end{array}\right.
\end{array}\right.
$$

Using the splitting method with respect to time (see [14]), on the time period $\Delta t$, we solve Eq. (9) with the time variable $\tau \in[0, \Delta t]$ by Eqs. (10), (11)

$$
\begin{aligned}
& \left\{\begin{array}{l}
\frac{\partial \widehat{C}}{\partial \tau}+\widehat{U}_{l_{j, i k}} \frac{\partial \widehat{C}}{\partial x_{j, i k}}-\eta \frac{\partial^{2} \widehat{C}}{\partial x_{j, i k}^{2}}-K \widehat{C}=0 \\
\lim _{x_{j, k} \rightarrow \pm 0} \widehat{C}=\left\{\begin{array}{l}
C_{\Omega j}, x_{j, i k}<0 \\
C_{\Omega_{l}}, x_{j, i k}>0
\end{array}\right.
\end{array}\right. \\
& \left\{\begin{array}{l}
\frac{\partial C^{*}}{\partial \tau}=\Phi \\
\left.C^{*}\right|_{\tau=0}=\left.\widehat{C}\right|_{\tau=\Delta t}
\end{array}\right.
\end{aligned}
$$

If $\Delta t$ small enough we have (see [14])

$$
\left.\left.C\left(x_{j, i k}, \tau\right)\right|_{\tau=\Delta t} \approx C^{*}\left(x_{j, i k}, \tau\right)\right|_{\tau=\Delta t} ; \quad \frac{\left.\partial C\left(x_{j, i k}, \tau\right)\right|_{\tau=\Delta t}}{\partial x_{j, i k}} \approx \frac{\left.\partial C^{*}\left(x_{j, i k}, \tau\right)\right|_{\tau=\Delta t}}{\partial x_{j, i k}} .
$$

If $K=0$ the solution of Eq. (10) (see [15] pp. 618-619) is

$$
\widehat{C}\left(x_{j, i k}, \tau\right)=R\left(x_{j, i k}, \tau\right)
$$

where

$$
R\left(x_{j, i k}, \tau\right)=A_{j}-B_{j} \operatorname{erf} \mathcal{C}\left(\frac{x_{j, i k}-\widehat{U}_{l_{j, i k}} \tau}{2 \sqrt{\eta \tau}}\right) ; A_{j}=\frac{C_{\Omega j}+C_{\Omega_{l}}}{2} ; B_{j}=\frac{C_{\Omega j}-C_{\Omega_{l}}}{2} .
$$

If $K \neq 0$, it is easy to check that: $\widehat{C}\left(x_{j, i k}, \tau\right)=e^{K \tau} R\left(x_{j, i k}, \tau\right)$ is the solution of (10).

The solution of Eq. (11) can be approximated by: $C^{*}\left(x_{j, i k}, \Delta t\right)=C^{*}\left(x_{j, i k}, 0\right)+\Phi \Delta t$. Using the initial condition $C^{*}\left(x_{j, i k}, 0\right)$ in this formula and thanks to (12)-(13) we get

$$
\begin{gathered}
\left.C(0, \tau)\right|_{\tau=\Delta t} \approx C^{*}(0, \Delta t)=e^{K \Delta t}\left(A_{j}-B_{j} \operatorname{erfc}\left(\frac{-\widehat{U}_{l_{j, i k}} \sqrt{\Delta t}}{2 \sqrt{\eta}}\right)\right)+\Phi \Delta t, \\
\left.\left.\frac{\partial C\left(x_{j, i k}, \tau\right)}{\partial x_{j, i k}}\right|_{x_{j, i k}=0, \tau=\Delta t} \approx \frac{\partial C^{*}\left(x_{j, i k}, \tau\right)}{\partial x_{j, i k}}\right|_{x_{j, i k}=0}=e^{K \Delta t} B_{j} \frac{e^{\frac{-\hat{u}_{j, i k}^{2} \Delta t}{4 \eta}}}{\sqrt{\eta \pi \Delta t}} .
\end{gathered}
$$


Using (7), (8), (16) and the formula of $B_{j}$ in Eq. (14) it is easy to see that the exchanging concentration flux $\frac{\partial C}{\partial \vec{n}_{j, i k}}$ from $j$-cell to $l$-cell and the other one $\frac{\partial C}{\partial \vec{n}_{l, i k}}$ from $l$-cell to $j$-cell have the same absolute values but not the same sign.

To get more exact value of the function $G(C) \vec{n}_{j, i k}$ (see (6)) we divide the value of time step $\Delta t$ by $m$ (in our model $m=10$ ). Then, using the formulas (15), (16) we get the approximation formula of $G(C) \vec{n}_{j, i k}$ as follows

$$
G(C) \vec{n}_{j, i k} \approx \frac{1}{m} \sum_{r=1}^{m} e^{K \frac{r}{m} \Delta t}\left[\begin{array}{c}
\widehat{U}_{l, i k} A_{j}-B_{j} \widehat{U}_{l, i k} \operatorname{erfc}\left(\frac{-\widehat{U}_{l, i k} \sqrt{\frac{r \Delta t}{m}}}{2 \sqrt{\eta}}\right) \\
-B_{j} \frac{e^{\frac{-\widehat{u}_{l, i k}^{2} \frac{r \Delta t}{m}}{4 \eta}}}{\sqrt{\frac{r \Delta t}{m}}} \sqrt{\frac{\eta}{\pi}}+\frac{1}{m} \sum_{r=1}^{m} \widehat{U}_{l, i k} \Phi \frac{r \Delta t}{m}
\end{array}\right] .
$$

Applying the approximation of $G\left(C_{t}\right) \vec{n}_{j, i k}$ given by (17) into the formula (5) the value $C_{\Omega j, t+\Delta t}$ is obtained. To get the good result of the concentration $C$, the time step $\Delta t$ needs to satisfy the Courant-Friedrichs-Lewy (CFL) condition.

\subsection{Improved approximation schema (IAS) for the estimation of $X=(h, u, v)^{T}$ and $C$}

The discrete schema calculating $X$ and $C$ can be briefly written by the following steps:

(i) The starting values of $X$ and $C$ are given at $N_{p}$ mesh points;

(ii) Calculate the values of $X$ and $C$ in $N$ cells by the same ways as in [3];

(iii) The values of $X=(h, u, v)^{T}$ and $C$ in $N$ cells are called by the values of the previous step;

(iv) Calculate $X=(h ; u ; v)^{T}$ by the schema in [11];

(v) On the cell boundaries $\gamma_{j}(j=1, \ldots, N)$, the function $G\left(C_{t}\right)_{l_{j, i k}} \vec{n}_{j, i k}$ involved in (5) is calculated by (17);

(vi) Calculate the function $C$ for the current step by (5);

(vii) Using the obtained values of $X=(h, u, v)^{T}$ and $C$ in current step at $N$ cell centers calculate $X, C$ in $N_{p}$ mesh points by the same ways as [3];

(viii) For the next step go to (iii).

\section{NUMERICAL TEST CASES FOR POLLUTION TRANSMISSION MODEL}

To test the numerical algorithm for a 2D pollution model, we will consider 2 test cases using data displayed in Tabs. 1 and 2. In these test cases, we consider the problems of water flows with constant velocity $U=(u, 0)$ in a channel with length $L$ and width $W$. We denote the gate-in of the channel by $\Gamma_{1}$, the gate-out is $\Gamma_{2}$ and the solid is $S_{w}$. The boundary conditions on $\Gamma$ are

$$
\left\{\begin{array}{l}
C=C_{i n} \text { on } \Gamma_{1} \\
C=C_{i n} \text { on } \Gamma_{1} \text { for the fist and the second test cases } \\
\frac{\partial C}{\partial n}=0 \text { on } S_{w} \text { for the second test cases }
\end{array}\right.
$$


The initial condition is $C(x, y, 0)=C$. In the first test case, we will test 1D model for concentration and then suppose that there is a dissolvable substance distributed in the channel only in the direction of $x$. The 2D model for concentration is tested by the second test case.

Table 1. Data of the channel

\begin{tabular}{|c|c|c|c|c|c|c|c|c|}
\hline Pr. & $W(\mathrm{~m})$ & $L(\mathrm{~m})$ & $K$ & $u(\mathrm{~m} / \mathrm{s})$ & $\eta$ & $C_{i n}(\mathrm{mg} / \mathrm{l})$ & $C_{0}(\mathrm{mg} / \mathrm{l})$ & Time step $(\mathrm{s})$ \\
\hline 1 & 200 & 10000 & 0 & 0.5 & 30 & Formula (19) & Formula (18) & 1 \\
\hline 2 & 2000 & 5000 & -0.0001 & 1 & 10 & Formula (25) & Formula (24) & 1 \\
\hline
\end{tabular}

Table 2. Data of the channel

\begin{tabular}{|c|c|c|c|c|}
\hline Pr. & $\Gamma_{1}$ & $\Gamma_{2}$ & $C_{\text {out }}(\mathrm{mg} / \mathrm{l})$ & $\frac{\partial C}{\partial n}$ on $\Gamma_{2}$ \\
\hline 1 & $x=-5000, y \in[0,200]$ & $x=5000, y \in[0,200]$ & Formula (20) & - \\
\hline 2 & $x=0, y \in[-1000,1000]$ & $x=5000, y \in[-1000,1000]$ & Formula (26) & - \\
\hline
\end{tabular}

\subsection{The first test case}

For this test case we suppose that $-L / 2 \leq x \leq L / 2$ and the concentration function $C$ is only depending on $x$ and $t$. In the initial moment the concentration $C(x, 0)$ has a discontinuity at $x=0$ (see [15]) such that

$$
C(x, 0)=C_{0}=\left\{\begin{array}{l}
C_{2} \text { if } x \in\left[-\frac{L}{2}, 0\right] \\
C_{1} \text { if } x \in\left[0, \frac{L}{2}\right] \\
C_{2} \text { when } x \rightarrow-0 \\
C_{1} \text { when } x \rightarrow+0
\end{array}\right.
$$

The values of $C_{1}$ and $C_{2}$ are: $C_{2}=30 \mathrm{mg} / 1, C_{1}=10 \mathrm{mg} / 1$. The boundary conditions on the gate-in $\Gamma_{1}$ and the gate-out $\Gamma_{2}$ are given by

$$
\begin{aligned}
& C_{\text {in }}(t)=A_{1}-A_{2} \operatorname{erfc}\left(\frac{-L / 2-u t}{2 \sqrt{\eta t}}\right), \\
& C_{\text {out }}(t)=A_{1}-A_{2} \operatorname{erfc}\left(\frac{L / 2-u t}{2 \sqrt{\eta t}}\right),
\end{aligned}
$$

with $A_{1}=\frac{C_{2}+C_{1}}{2}$ and $A_{2}=\frac{C_{2}-C_{1}}{2}$. The equation and boundary and initial conditions of the first test are 


$$
\left\{\begin{array}{l}
\frac{\partial C}{\partial t}+u \frac{\partial C}{\partial x}-\eta \frac{\partial^{2} C}{\partial x^{2}}=0 \text { in } \Omega \\
C=C_{\text {in }}(t) \text { on } \Gamma_{1} \text { (see formula (19)) } \\
C=C_{o u t}(t) \text { on } \Gamma_{2} \text { (see formula (20)) } \\
C(x, 0)=C_{0}(t) \text { (see formula (18)) }
\end{array}\right.
$$

The exact solution of Eqs. (21) is (see [15] pp. 618-619): $C_{1}(x, t)=A_{1}-A_{2} \operatorname{erfc}\left(\frac{x-u t}{2 \sqrt{\eta t}}\right)$. Comparing the exact solution with the solutions obtained by the normal differential implicit schema (NDIS) (see [3]) and the IAS in the item 3.2 gives that the simulation solution is closer to the exact solution (see Fig. 2).
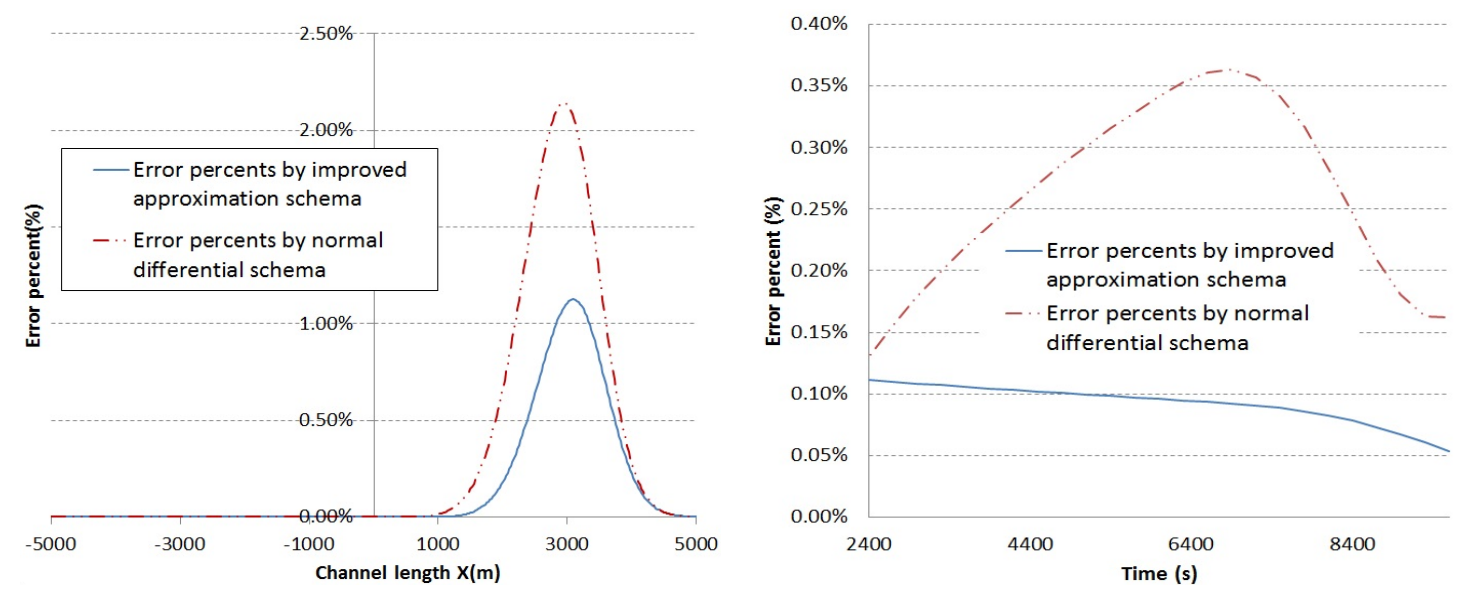

Fig. 2. The first test case of Section 4: Error percentage for simulated and exact solutions by the improved approximation schema and by the normal differential implicit schema (left); Mean absolute errors of concentration in percentage of mean concentration (right)

At time $5400 \mathrm{~s}$, the comparison of error percentages between the simulated solutions, using the IAS then by the NDIS and the exact solution (see [3]) is displayed in the left side of Fig. 2. The mean error percentages of concentration simulations by time period of the two schemes are shown in the right side of Fig. 2. On this figure we see that the mean concentration error of the model using the IAS is better than the one obtained by the NDIS and less than $0.125 \%$.

\subsection{The second test case}

The concentration of pollutant for this test case verifies

$$
\frac{\partial C}{\partial t}+u \frac{\partial C}{\partial x}-\eta\left(\frac{\partial^{2} C}{\partial x^{2}}+\frac{\partial^{2} C}{\partial y^{2}}\right)=K C, \text { in } \Omega .
$$

The exact solution of this equation is (see $[16,17])$

$$
C_{2}(x, y, t)=\frac{M}{4 \pi \eta t} e^{-\frac{(x-u t)^{2}+y^{2}}{4 \eta t}+K t},
$$


where $M$ is a constant. In this case, the values of the initial moment $t_{0}$ and $M$ are: $t_{0}=$ $1000 \mathrm{~s}$ and $M=10^{3} \mathrm{mg} / \mathrm{l}$. The initial condition of the concentration is given by

$$
C_{0}(x, y)=\frac{M}{4 \pi \eta t_{0}} e^{-\frac{\left(x-u t_{0}\right)^{2}+y^{2}}{4 \eta t_{0}}+K t_{0}} \text {. }
$$

The concentration boundary conditions on the gate in $\Gamma_{1}$ and the gate out $\Gamma_{2}$ are given by the formulas (25) and (26), respectively,

$$
\begin{gathered}
\left.C(x, y, t)\right|_{\Gamma_{1}}=C(0, y, t)=\frac{M}{4 \pi \eta\left(t_{0}+t\right)} e^{-\frac{u^{2}\left(t+t_{0}\right)^{2}+y^{2}}{4 \eta\left(t_{0}+t\right)}+K\left(t_{0}+t\right)}, \\
\left.C(x, y, t)\right|_{\Gamma_{2}}=C(L, y, t)=\frac{M}{4 \pi \eta\left(t_{0}+t\right)} e^{-\frac{\left(L-u\left(t+t_{0}\right)\right)^{2}+y^{2}}{4 \eta\left(t_{0}+t\right)}+K\left(t_{0}+t\right)} .
\end{gathered}
$$

Data for this test case are shown in Tabs. 1 and 2. For the second test case we have the following governing equations

$$
\left\{\begin{array}{l}
\frac{\partial C}{\partial t}+u \frac{\partial C}{\partial x}-\eta\left(\frac{\partial^{2} C}{\partial x^{2}}+\frac{\partial^{2} C}{\partial y^{2}}\right)=K C \text { in } \Omega \\
C=C_{i n} \text { on } \Gamma_{1}(\text { see formula }(25)) \\
C=C_{\text {out }} \text { on } \Gamma_{2} \text { (see formula (26)) } \\
\frac{\partial C}{\partial n}=0 \text { on } S_{w} \\
C(x, y, 0)=C_{0}(\mathrm{x}, \mathrm{y}) \text { (see formula (24)) }
\end{array}\right.
$$
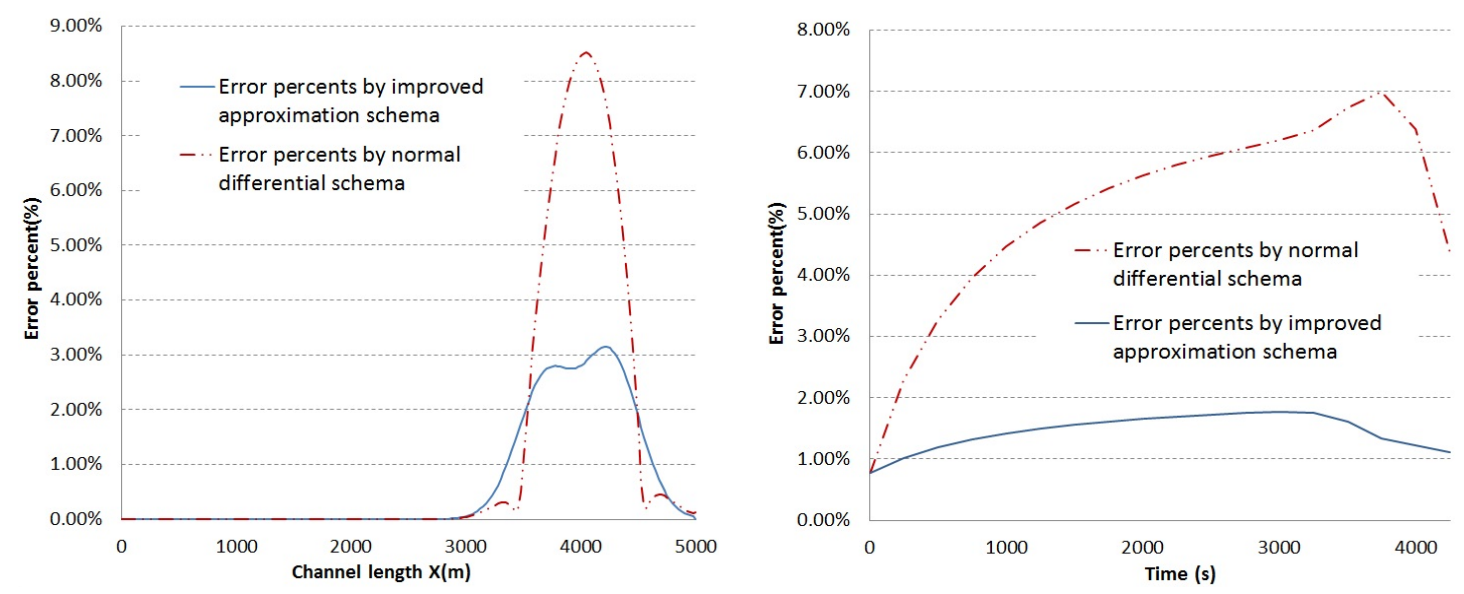

Fig. 3. The second test case of Section 4: Error percents of simulations and exact solution by improved approximation schema and by the normal differential implicit schema in the middle of the channel $(y=0)$ (left); Mean absolute errors of concentration in percentage of mean concentration (right)

At time $3000 \mathrm{~s}$ the left side of Fig. 3 describes the comparison of error percentage between simulated concentration $C(x, y, t)$ of $(27)$ and the exact solution $C_{2}\left(x, y, t+t_{0}\right)$ 
of (23) by the IAS and by the NDIS (see [3]), respectively. The mean absolute error percentage of concentration of the two schemes are shown in the right side of Fig. 3 . On this figure we see that the error percentage of the model is smaller than the one we get by using NDIS and less than $1.8 \%$.

The mean absolute errors of 2 test cases are presented in the right sides of Figs. 2, 3 and less than $1.8 \%$. The results obtained in the in 2 test cases show that the simulation results of this model using the IAS are closer to the exact solutions than what we get using the NDIS and furthermore the numerical solutions are stable.

\section{CONCLUSIONS}

The numerical model solving 2D pollution water model is used with HLL Riemann solver schema for solving water velocity (see [11]) and improved by the new concentration approximation schema when we solve the pollution equation. The model is tested with 2 cases. The following results are obtained:

- Two concentration test cases are studied with the new improved concentration approximation schema. The first test case is considered by the pollution transmission problem of a dissolvable substance distributed in the channel only by $x$-direction. In this test case with discontinuity at $x=0$ the solution has a fast evolution.

- The second test case is tested for $2 \mathrm{D}$ pollution problem. The results show that the model is working well with the parameters of concentration equation changing in large intervals. The percent errors between the exact solutions and the simulations using the new approximation schema are less than the one using the normal differential implicit schema. It shows the advantage of the improved concentration approximation.

These results demonstrate that the presented algorithm is an improved tool for concentration calculations, in the case of 2D water pollution problems.

\section{ACKNOWLEDGMENT}

The authors acknowledge the support by the NAFOSTED's 107.03-2015.31 funds. This paper was partly written while one of the authors (Prof. Le Dimet) was invited at Florida State University by Prof. M. Y. Hussaini.

\section{REFERENCES}

[1] D. Toro, C. Winfield, and R. B. Ambrose. QUAL2K. Brown and Barnwell, Water Quality Analysis Simulation Program, United States Environmental Protection Agency, (1987).

[2] T. T. Ha and N. H. Phong. 2D-Model of contaminant water transmission processes and numerical simulation on a natural lake. Vietnam Journal of Mechanics, 32, (3), (2010), pp. 157-166. doi:10.15625/0866-7136/32/3/304.

[3] T. H. Tran, D. T. Pham, H. V. Lai, and H. P. Nguyen. Water pollution estimation based on the 2D transport-diffusion model and the singular evolutive interpolated Kalman filter. Comptes Rendus Mécanique, 342, (2), (2014), pp. 106-124. doi:10.1016/j.crme.2013.10.007.

[4] W. Rauch, M. Henze, L. Koncsos, P. Reichert, P. Shanahan, L. Somlyódy, and P. Vanrolleghem. River water quality modelling: I. State of the art. Water Science and Technology, 38, (11), (1998), pp. 237-244. 
[5] C. P. Dullemond and J. A. Hydrodynamics II: Numerical methods and applications. University of Heidelberg Summer Semester, (2007).

[6] T. Stocker. Introduction to climate modeling. Springer-Verlag Berlin Heidelberg, (2011).

[7] C. Licht, T. T. Ha, and Q. P. Vu. On some linearized problems of shallow water flows. Differential and Integral Equations, 22, (3/4), (2009), pp. 275-283.

[8] P. A. Sleigh, P. H. Gaskell, M. Berzins, and N. G. Wright. An unstructured finite-volume algorithm for predicting flow in rivers and estuaries. Computers $\mathcal{E}$ Fluids, 27, (4), (1998), pp. 479508. doi:10.1016/s0045-7930(97)00071-6.

[9] E. F. Toro. Riemann problems and the WAF method for solving the two-dimensional shallow water equations. Philosophical Transactions of the Royal Society of London A: Mathematical, Physical and Engineering Sciences, 338, (1649), (1992), pp. 43-68. doi:10.1098/rsta.1992.0002.

[10] X. Ying, J. Jorgeson, and S. S. Wang. Modeling dam-break flows using finite volume method on unstructured grid. Engineering Applications of Computational Fluid Mechanics, 3, (2), (2009), pp. 184-194. doi:10.1080/19942060.2009.11015264.

[11] N. H. Phong, T. T. Ha, F. X. Le Dimet, and D. N. Hai. A wind-driven hydrodynamic and pollutant transport model with application of HLL Riemann solver schema. In International Conference on Engineering Mechanics and Automation (ICEMA 3), (2014), pp. 146-155.

[12] D. Ambrosi. Approximation of shallow water equations by Roe's Riemann solver. International Journal for Numerical Methods in Fluids, 20, (2), (1995), pp. 157-168. doi:10.1002/fld.1650200205.

[13] G. Chen, H. Tang, and P. Zhang. Second-order accurate Godunov scheme for multicomponent flows on moving triangular meshes. Journal of Scientific Computing, 34, (1), (2008), pp. 64-86.

[14] G. I. Marchuk and J. Ruzicka. Methods of numerical mathematics, Vol. 2. Springer-Verlag, New York, (1975).

[15] C. Hirsch. Numerical computation of internal and external flows. Vol. 2: Computational methods for inviscid and viscous flows. John Wiley \& Sons, (1990).

[16] B. Hunt. Dispersive sources in uniform ground-water flow. Journal of the Hydraulics Division, 104, (1978), pp. 75-85.

[17] T. Karvonen. Pollutant transport in rivers. Department of Civil and Environmental Engineering, Helsinki University of Technology, (2002). 